\title{
Algorithm for Tribokinetic Modeling Tests of Triboconjuction Materials for Industrial Products
}

\author{
O.I. Bogdanovycha, T.M.A. Al-Quraan, ${ }^{\text {,* }, ~ V . V . ~ T o k a r u k a, ~ J . S . ~ H a d d a d c ~}$ \\ aNational Aviation University- Aircraft Airworthiness Retaining Department, 03058 Kiev, Ukraine, \\ ${ }^{b}$ Al-Balqa Applied University, Ma'an College -Technical Sciences Department, 19117 Al-Balqa , Jordan, \\ cAl-Balqa Applied University, Faculty of Engineering Technology, Mechanical Engineering Department, 11134 \\ Amman, Jordan.
}

Keywords:

Friction

Wear resistance

Tribokinetic tests

Kinetic characteristics

Activation energies

\section{* Corresponding author:}

Tareq M.A. Al-Quraan

E-mail: tarjor999@gmail.com

Received: 2 October 2020

Revised: 24 November 2020

Accepted: 25 February 2021

\begin{abstract}
A B S T R A C T
An experimental-calculation procedure for determining the kinetic and energy activation criteria for assessing the wear resistance and compatibility of triboconjunction materials using the kinetic three-stage triboreaction is presented. The developed method for tribo-kinetic tests to determine the kinetic characteristics (order and rate constants) and activation energies for all three stages of the triboreaction was tried experimentally for steels ShKh-15 and 45, aviation fuel RT, aviation hydraulic oil AOH-10 and aviation oil MK-8 added with oleic acid. The conducted tribo-kinetic tests established kinetic and activation energy criteria for assessing the wear resistance of ShKh-15 steel and antiwear properties of RT and AOH-10 lubricants, as well as kinetic criteria for assessing the wear resistance of steel 45 and antiwear properties of $M K-8$ with oleic acid. Also, using the developed method for tribokinetic tests, the kinetic and activation energies of ShKh-15 in long-term storage aviation fuels "TS-1" and "TS-1*", kinetic criteria and activation energy for the second stage of the triboreaction, chemical modification ShKh-15 in the medium of "TS-1", as well as the established kinetic and activation energy of the triboreaction, that is, surface destruction, in fact, wear of steel 45 during reciprocating motion.
\end{abstract}

(C) 2021 Published by Faculty of Engineering

\section{INTRODUCTION}

Acceleration of scientific and technological progress, as applied to industry, provides an increase in the quality, reliability, efficiency and productivity of machines, mechanisms, equipment and other industrial products. The solution of this problem relative to friction units of machines and mechanisms is impossible without unified modeling tests and the accumulation of the data bank of their results. At the same time, the criteria for assessment of the processes under study are of particular importance, since the effectiveness of the use of any algorithm for tribological modeling tests fundamentally depends on the invariance and constructiveness of the selected criteria for estimation of the studied processes. In this 
regard, the development of fundamental invariant energy criteria for assessing the wear resistance and compatibility of structural and lubricating materials and the corresponding methods for obtaining unified data has become extremely important in tribological engineering.

As such criteria, such kinetic characteristics have been studied as constants of the rate and activation energy for the determining processes in tribo-systems.

The basic requirements for these criteria are their invariance (independence of the loading conditions) and commonality for structural and lubricating materials, which makes it possible to select their compatibility (optimal combinations) [1].

The aim of the work is to develop an algorithm (methodology) for tribological modeling tests of structural and lubricating materials of triboconjunctions using such kinetic characteristics as constants of the rate and activation energy of the determining processes (significantly slow) in tribo-systems.

\section{PROBLEM STATEMENT}

Today most physical processes are known to mankind in the process of friction, such characteristics should be integral, energy material scientific for triboconjunction materials. For example, in the structural-energy theory of friction and wear and the theory of structural adaptability of triboconjunction materials, the following characteristics are used: the specific work of destruction $-A_{p}$ [2], energy consumption of the friction system on the thermal index - «EFSq » [2], critical friction value $-A_{c r}$ [2], critical (boundary) density of internal energy $-U^{*}$, (for abrasive wear) [3], surface energy or surface tension - $\gamma$ [4], work of the electron output WEO [4], exoelectronic emission - EEE [4], contact potential difference $U_{C P D}$ [4]. At the meantime, the energy characteristics of wear resistance characterize the processes of surface destruction, substantiate the evaluation of friction conditions, the choice of materials for triboconjunctions, their combination within normal wear (without damage) determine the limits of transition to damage.

The indicated criteria are not direct characteristics of the wear resistance of the triboconjunction materials. Thus, the most commonly used, best-studied and experimentally tested criterion - the specific work of surface fracture (the ratio of frictional force to wear) which is the imaginary characteristic of wear resistance and depends not only on the surface strength of the material, but also on the ability of the tribo-system to dissipate energy. Usage of surface energy $\gamma$ is too complicated due to the lack of reliable data on the magnitude $\gamma$ for pure metals, and moreover for alloys in real conditions [4].

Energy compatibility assessment criterion which is either surface energy or surface tension $\gamma$, or work of the output of the electron etc., as well as the criteria for wear resistance, are not direct compatibility characteristics. In addition, there is no reliable data $\gamma$ even for pure metals, $\gamma$ and different values of electron output work for different faces of the crystal [4].

The most direct, quantitative, structural-energy (material-science), kinetic (physical-chemical) characteristic that is useful for assessing wear resistance of triboconjunction materials is the activation energy of surface deterioration (wear) $E_{p}$, which characterizes the potential barrier to the implementation of elementary acts of destruction [5]. At the same time, in the range of structural adaptability of triboconjunction materials $E_{p}$ must be invariant $[5,6]$, which can be used as a criterion for assessment the anti wear properties of the lubricants [7]. This, in contrast to the existing variety of criteria for assessing wear resistance, is regulated by GOST 9490-75 and GOST 23.002-78, allows usage of the $E_{p}$ as a single universal criterion for evaluation of tribological properties of triboconjunction materials.

Versatility $E_{p}$ is confirmed by its application as a criterion for evaluating various physical processes. An example is the application of activation energy $U$ as functions of resistance to deformation of a plastic deformable metal sample. In the meantime $U$ which describes the processes of rearrangement of atoms in a crystal lattice, determines the degree of violation of the order that causes deformation [8]. Despite the coincidence of values of activation energy $U$ of diffusion and self-diffusion [9], as well as confirmation of equality $U \cong 20 R T_{M p}$, where $T_{M p}$ is Melting point [10], and this conclusion does not provide confident results due to the low accuracy of the degree of violation of the order of the crystal lattice. 
However, even the correct choice of criteria for assessing the wear resistance and compatibility of triboconjunction materials does not guarantee the correct determination of wear resistance and compatibility of these materials.

The correct choice of the criteria for assessing wear and compatibility is a necessary condition, but not a single one. An adequate condition for the correct assessment of wear resistance and compatibility of triboconjunction materials is the correct method for assessment of wear resistance and compatibility of these materials.

\section{RESEARCH'S CONSIDERATION}

Nowadays three methods for evaluating the activation energy are known: 1) Graphic [9,10,11, 12,15]; 2) Extrapolation [16]; 3) Kinetic $[7,13,14,17,18]$. In the first method the value of $E^{d}$ depends on the scale of the coordinate system. Therefore $E^{d}$ can only be used in comparative trials [18]. In the second method of meaning $E^{d}$ depends on the subjective qualities of the researcher since extrapolation is an approximation of the values $f_{(x)}$ in points $x$ lying outside the segment $\left(x_{0}, x_{n}\right)$ by their values at points $x_{0}<x<\ldots<x_{n}$. However, the best and the most precise technique is the third one -kinetic, where the value $E^{d}$ is determined by the laws and the equation of chemical kinetics, when first the order of the chemical reaction determined, then the constant of the rate of this reaction at least at two temperatures reaction are either determined and finally $E^{d}$ is calculated using the Arrhenius equation [7]. This allows to calculate the accuracy of the determination of kinetic parameters (order and rate of reaction) and $E_{p}$ according to statistical laws and equations, which considerably increases the constructivity of these criteria and confirms the possibility of their use as criteria for the assessment of wear resistance and compatibility of triboconjunction materials. However, the kinetic technique was developed on the basis of a kinetic, three-stage model of normal mechanical and chemical wear, for the description of which the following scheme of the tribological reaction (tribo-reaction) is used:

$$
R B \stackrel{A}{\longrightarrow} M e_{x 1}(R B)_{y 1}^{*} \stackrel{M}{\longrightarrow} M e_{x 2} B_{y 2}^{*} \stackrel{D}{\longrightarrow} M e_{x 3} B_{y 3}^{*} \stackrel{D}{\longrightarrow} M e^{o}
$$

Where $R B$ - lubricant that takes part in a threeway reaction: in the simplest case only the effect of the active component of the lubricant-additive can be considered, therefore RB is an active component of the lubricant (additive), $R$ hydrocarbon radical additive, B - active component of the additive, $M e^{o}$ - juvenile surfaces of contact surfaces of triboconjunction materials; $X_{i}, Y_{i}$ - partial coefficient of components, which are analogs of stoichiometric coefficients in true chemical reaction.

The following active complexes of a triboreaction are marked by the asterisk:

$M e_{x 1}(R B)_{y 1}^{*}$ - Adsorption complex formed as a result of the adsorption of the lubricant $R B$ on the juvenile sections of the surfaces of structural triboconjunction materials $M^{o}$. This complex is active in relation to the next stage - chemical modification;

$M e_{x 2} B_{y 2}^{*}$ - modified (dissipative secondary) structures, active in relation to the stage of surface destruction $P$;

$M e_{x 3} B_{y 3}^{*}$ - A complex of surface destruction (products of wear), in essence - a product of a tribo-reaction.

As it can be seen from tribo-reaction (1), only the first stage adsorption $A$ is considered as a reverse, i.e. two-way one. Other stages are considered to be substantially reversible, i.e. one-way ones. The juvenile areas of contacting structural materials are both an initial reactive component and a catalytic tribo-reaction, which accelerates the course of the first stage $A$. In this case, the total area of juvenile sections, which is the concentration $M^{o}$ in normal (stationary) wear it remains constant [19], while the amount of output materials is constantly decreasing as a result of wear. The amount of lubricating and construction materials is assumed to be sufficiently large and their concentration does not depend on the duration of the triboreaction. Otherwise, it is necessary to use absolute values rather than concentrations.

According to the above-described kinetic threestage model of normal mechanical and chemical deterioration, it is necessary to develop an experimental calculation method for evaluating the kinetic characteristics and activation energies of all three stages of a three-way reaction. 
Paradoxically it is necessary to determine firstly the kinetic characteristics and the activation energy of $E^{d}$ - a third, the last stage of the threeaction that is, actually a wear itself as the result of conducting tribo-kinetic trials of materials triboconjunctions.

The ball segments $V_{w}$ are chosen by means of measuring the diameter of the spots of wear of three balls and the amount of wear is calculated. After conducting similar tribo-kinetic tests at 4-5 values of the duration of such tests $t$, we construct the dependency schedules $\lg V_{w}$ from the duration of the test $t$. The straight lines of such graphs indicate the first order of the investigated stage (in this case, the third stage of the tribo reaction), i.e. $N^{d} \cong 1$ while a tangent of an angle of inclination to the abscissa axis of a graph determines the speed constants of the investigated stage, in this case $K_{p}$. However, the graphical evaluation and the values of the order and the rate constants of the studied tribo-reaction steps gives approximate results only. Therefore, more accurate values of these kinetic characteristics should be determined analytically, i.e. determine using the required formulas. For example, the order of the study stage, that is the third one is calculated by the following formula (2):

$$
N^{d}=\frac{\lg \frac{W_{2}}{W_{1}}}{\lg \frac{V_{w t_{i+1}}}{V_{w t_{i}}}}
$$

Where $\mathrm{W}_{1}$ and $\mathrm{W}_{2}$ are the speeds of stage III i.e. the wear determined for the intervals of tribokinetic tests calculated via formulas (3) and (4):

$$
\Delta t=t_{i+1}-t_{i}
$$

Where

$$
W_{1}=\frac{V_{w t_{i+1}}-V_{w t_{i}}}{\Delta t}
$$

Where $V_{w t_{i+1}}$ and $V_{w t_{i}}$ are the wear volumes of three balls during tribo-kinetic tests at the duration of $t_{i+1}$ and $t_{i}$ respectively.

The order of stage III can be calculated by two ways for each:

1) Calculation for each interval $\Delta t$ and then calculate the arithmetic mean $\mathrm{N}_{\mathrm{i}}$.
2) Calculation of $\Delta t$ start and end intervals while, $N_{d}$ is calculated during the entire duration of tribo-kinetic tests.

The rate constant of stage III, wear was calculated using the difference $V_{w}$ in the interval $\Delta t$ by the formula (5):

$$
K^{d}=\frac{\Delta V_{w}}{\Delta \mathrm{t} V_{w \cdot m}}=\frac{\mathrm{W}}{V_{w \cdot m}}
$$

Where $V_{\text {w.m. }}$ is the average value of $V$ at the beginning and end of the interval $\Delta t$.

By setting a minimum 4 value of $K^{d}$, it is possible to perform statistical processing of the obtained results, i.e. to calculate the average values of $K^{d}$, the confidence interval of the estimation $K^{d-} \Delta K^{d}$, and $Z_{c}$ - the coefficient of variation of that estimation.

Repeating tribo-kinetic tests according to the above procedure at another value of temperature different from $T_{1}$, according to the Arrhenius equation we calculate the activation energy of the third stage of the $T R$ - the actual wear of the tribo- materials.

In addition, a following formula (6) is proposed by an author for calculating the area of secondary structures participating in the $T R\left(\mathrm{~S}_{\mathrm{ss}}\right)$ [20] and as a consequence, the calculation of the relative area of the secondary layers,

$$
\delta=\frac{S_{\mathrm{ss}}}{\mathrm{S}_{\mathrm{f}}}
$$

Where $S_{f}$ the area of contact, i.e., the contact area of the three spots of allowed to calculate the kinetic characteristics $\left(N m, K^{m}\right)$ and activation energy of the second stage of $T R$ chemical modification $\left(E^{M}\right)$ using the formula (7):

$$
S_{s s}=\mathrm{S}_{\mathrm{f}}-\frac{\Delta V_{w \cdot m}}{\Delta t h_{s s} K^{d}}
$$

Where $\Delta V_{w . m}=V_{w t_{i+1}}-V_{w t_{i}}$ is the wear volume of three balls in the interval $\Delta t=t_{i+1}-t_{i}$ in respectively; $h_{c c}$ is the thickness of the secondary structures or the thickness of the chemical modification. This value is variable: with the increment of the duration of tribo-kinetic tests $h_{\phi c}$ decreases. 
Kinetic characteristics of the first stage of $T R(N a$, $\mathrm{Ka}$ ) and the activation energy can be determined by performing tribo-kinetic tests using the specified algorithm with a chemical-active substance, i.e. an additive, such as oleic acid [21], which allows to determine the kinetic parameters and the activation energy of tribosorption on the activation of a chemical substance.

These tribo-kinetic tests were performed at a single fixed temperature $T_{1}$. If similar tribokinetic tests are performed under a different temperature $T_{2}$ different from $T_{1}$, then the values of similar kinetic criteria $N$ and $K$ of the three stages of $T R$ are obtained. Knowing the value of $K$ at two temperatures $T_{1}$ and $T_{2}$, the activation energies of these stages are calculated according to the Arrhenius formula (8):

$$
E=\frac{R T_{1} T_{2}}{T_{1}-T_{2}} \ln \frac{K_{2}}{K_{1}}=\frac{1,9144 T_{1} T_{2}}{T_{2}-T_{1}} \lg \frac{K_{2}}{K_{1}} \times 10^{-2}
$$

Where $K_{1}$ and $K_{2}$ are the rate constants at temperatures $T_{1}$ and $T_{2}$ respectively, 1.9144 is the product of the gas constant $\mathrm{R}=$ $(8.3143 \pm 0.0012) \times 10^{3} \mathrm{j} / \mathrm{K} \cdot \mathrm{mol}$ by the module of conversion of natural logarithms to decimals, which is equal to 2.3056 .

In addition, in case there is a possibility to calculate the wear amount of the moving element of the triboconjunction, then the fourth value of activation energy of the value $E_{d}^{d_{d}}\left(E^{a}, E^{m}\right.$, $E_{n d}$ and $E_{d_{d}}$ ) is obtained. Therefore, the four values of $E$ constitute the matrix criterion for evaluating the durability and compatibility of tribomaterials

$$
\left|\begin{array}{llll}
E^{a} & E^{M} & E_{n d}^{d} & E_{d}^{d} \\
E_{1}^{a} & E_{1}^{M} & E_{n d 1}^{d} & E_{d 1}^{d} \\
E_{2}^{a} & E_{2}^{M} & E_{n d 2}^{d} & E_{d 2}^{d} \\
E_{3}^{a} & E_{3}^{M} & E_{n d 3}^{d} & E_{d 3}^{d} \\
E_{\mathrm{n}}^{a} & E_{n}^{M} & E_{n d n}^{d} & E_{n d n}^{d}
\end{array}\right|
$$

where $E_{d_{n d}}$ and $E_{d}$ are the activation energies values of the materials surface fracture for the both fixed and movable tribocoupling elements, respectively; " $A$ ", " $m$ " and " $d$ " are the activation energies of tribo adsorption, chemical modification, and surface destruction respectively, i.e. the actual wear of the tribo coupling elements of both mobile and stationary ones.

The investigation of tribo-kinetic study of the tribocoupling materials was carried out experimentally on two friction machines UPS-01 and MT-1 (Figure 1) [21], for steel ShKh 15 (an analogue of EU 100Cr6 and US 52100 steels) and steel 45 (analogue of EU C45 and US 1045 steels), RT aviation fuel (a certified analogue of Jet A-1 aviation kerosene), $\mathrm{AOH}-10$ hydraulic aviation oil, MK-8 aviation oil with the addition of oleic acid.

The conducted tribo-kinetic research has established kinetic $\left(N^{p}, K^{p}, N^{m}, K^{m}\right)$ and energyactivation $\left(E^{d}, E^{M}\right)$ criteria for evaluation of ShKh15 wear resistance and anti-wear properties of $\mathrm{RT}$ and $\mathrm{AOH}-10$, as well as kinetic $\left(\mathrm{N}^{a}, \mathrm{~K}^{\mathrm{a}}, \mathrm{N}^{\mathrm{m}}, \mathrm{K}^{\mathrm{m}}\right.$, $\mathrm{N}^{\mathrm{p}}, K^{\mathrm{d}}$ ) criteria for assessment of the wear resistance of steel 45 and the anti-wear properties of MK-8 with the addition of oleic acid $[20,21]$.

Later, kinetic characteristics $\left(N^{d}, K^{d}\right)$ were established using the developed tribo-kinetic test procedure as well as energy-activation wear ones $\left(E^{d}\right)$ of ShKh 15 steel in the medium of long-term storable aviation kerosene TS-1 and TS- ${ }^{*}$ (analogues of Jet A-1 fuel) [20, $22,23]$, then -kinetic characteristics $\left(N^{M}, K^{M}\right)$ and activation energy of the second stage of $T R$ - chemical modification $E^{M}$ of ShKh 15 in the medium of TS-1 kerosene $[23,24,25]$, as well as kinetic ones $\left(\mathrm{N} d, K^{\mathrm{d}}\right)$ and activation energy of $T R$ - surface destruction that is actually the wear $E^{d}$ [27] of steel 45 during reciprocating motion.

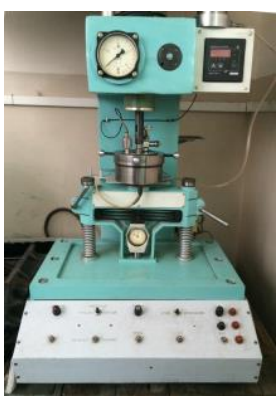

(a)

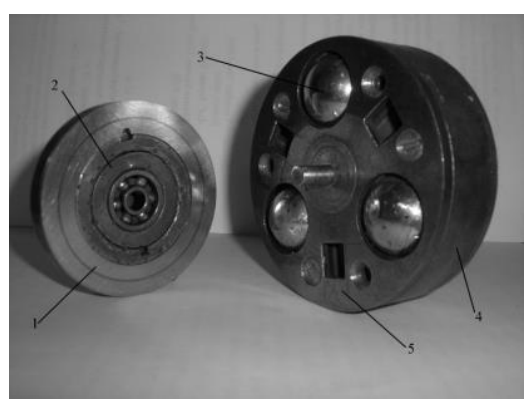

(b)
Fig. 1. (a) General view of the UPS-01, (b) A pair of friction, 1-Disc sample, 2-Disc sample holder, 3-Ball, 4- Separator, 5-Pin washer. 
Table 1. The Kinetic characteristics and activation energy of surface destruction of $E_{p}$ of steel ShKh 15 in longterm storage of TS-1 aviation fuel.

\begin{tabular}{|c|c|c|c|c|c|c|c|}
\hline $\begin{array}{l}\text { Axial } \\
\text { load } \\
P, N\end{array}$ & $\begin{array}{l}\text { Temperature } \\
T, K^{0}\end{array}$ & $\begin{array}{l}\text { Sequential } \\
\text { wear } \\
\text { reactions }\end{array}$ & $\begin{array}{l}\text { Time intervals } \\
\Delta t \text {, for which it } \\
\text { was calculated } \\
\Delta t \times 10^{-3}, c^{-1}\end{array}$ & $\begin{array}{l}\text { Value } K^{P} \\
\text { for } \\
\text { intervals } \\
\Delta t, K^{P} \times 10^{-3} \text {, } \\
c^{-1}\end{array}$ & $\begin{array}{l}\text { Average } \\
\text { values } \\
K^{P}, \\
K^{P} \times 10^{-3}, \\
c^{-1}\end{array}$ & $\begin{array}{l}\text { The coefficient } \\
\text { of estimate } \\
\text { variation, } K^{P} \text {, } \\
W_{K} \%\end{array}$ & $\begin{array}{l}\text { Value of } \\
\text { wear } \\
\text { activation } \\
\text { energy, } E_{p} \text {, } \\
\mathrm{kJ} / \mathrm{mol} \\
\end{array}$ \\
\hline \multirow{2}{*}{98,1} & 303 & $\begin{array}{l}1 \\
1\end{array}$ & $\begin{array}{c}3,6-3 \\
4,2-3,6 \\
4,8-4,2\end{array}$ & $\begin{array}{l}1,2094 \\
0,9927 \\
1,0595\end{array}$ & 1,0872 & 10,2 & \multirow{2}{*}{19,581} \\
\hline & 333 & $\begin{array}{l}1 \\
1\end{array}$ & $\begin{array}{c}2,4-1,8 \\
3-2,4 \\
3,6-3 \\
\end{array}$ & $\begin{array}{l}1,9868 \\
2,4038 \\
2,1791 \\
\end{array}$ & 2,1899 & 6,7 & \\
\hline
\end{tabular}

Table 2. The Kinetic characteristics of surface fracture of ShKh 15 steel in medium of TS-1 jet fuel.

\begin{tabular}{|c|c|c|c|c|c|c|c|c|}
\hline \multicolumn{2}{|l|}{ Value } & \multirow[b]{2}{*}{$\begin{array}{l}\text { Time } \\
\text { intervals } \\
\Delta \mathrm{t} \text {, for } \\
\text { which it } \\
\text { was } \\
\text { calculated } \\
\Delta \mathrm{t} \times 10^{-3}, \mathrm{c}^{-1} \\
\end{array}$} & \multirow[b]{2}{*}{$\begin{array}{l}\begin{array}{l}\text { Value } K^{P} \\
\text { for }\end{array} \\
\text { intervals } \\
\Delta t \text {, } \\
K^{P} \times 10^{-3}, \\
C^{-1}\end{array}$} & \multirow{2}{*}{$\begin{array}{l}\text { Average } \\
\text { values } \\
\mathrm{K}^{\mathrm{P}}, \\
K^{P} \times 10-3, \\
\mathbf{c}^{-1}\end{array}$} & \multirow[b]{2}{*}{$\begin{array}{l}\text { Trust } \\
\text { interval of } \\
\text { evaluation } \\
K^{P}, \pm \Delta K^{P} \times \\
10^{-3}, \mathbf{c}^{-1}\end{array}$} & \multirow{2}{*}{$\begin{array}{l}\text { Value } K^{\mathrm{P}} \\
\text { installed } \\
\text { graphically }\end{array}$} & \multirow[b]{2}{*}{\begin{tabular}{|l|} 
The \\
variation \\
coefficient \\
of \\
estimation, \\
$K^{P}, W_{K} \%$ \\
\end{tabular}} & \multirow[b]{2}{*}{$\begin{array}{l}\text { Value of } \\
\text { wear } \\
\text { activatio } \\
\text { n energy, } \\
E_{p}, \mathrm{~kJ} / \\
\mathrm{mol}\end{array}$} \\
\hline $\begin{array}{l}\text { Axial } \\
\text { load } \\
P, \mathrm{~N}\end{array}$ & $\begin{array}{l}\text { Temperature } \\
T, K^{o}\end{array}$ & & & & & & & \\
\hline \multirow{2}{*}{9,81} & 303 & $\begin{array}{l}2,4-1,8 \\
3,0-2,4 \\
3,6-3,0\end{array}$ & 0,4872 & 0,4897 & 0,0285 & $\operatorname{tg} 240=0,4452$ & 8,6749 & \multirow{2}{*}{19,4219} \\
\hline & 333 & $\begin{array}{l}2,4-1,8 \\
3,0-2,4 \\
3,6-3,0\end{array}$ & $\begin{array}{l}1,0241 \\
0,8878 \\
1,0305\end{array}$ & 0,9808 & 0,0422 & $\operatorname{tg} 440=0,9657$ & 8,2176 & \\
\hline
\end{tabular}

Table 3. The energy of activation of two stages of triboreaction for some fuels and lubricants.

\begin{tabular}{|c|c|c|c|c|c|}
\hline $\begin{array}{l}\text { Fuels and } \\
\text { lubricants }\end{array}$ & $\begin{array}{l}\text { Construction } \\
\text { material }\end{array}$ & $\begin{array}{l}\text { Stage of } \\
\text { triboreaction }\end{array}$ & $\begin{array}{l}\text { Energy of } \\
\text { activation, } \\
\mathrm{kJ} / \mathrm{mol}\end{array}$ & $\begin{array}{l}\text { Coefficient of } \\
\text { variation } W, \%\end{array}$ & $\begin{array}{l}\text { Direction of } \\
\text { friction }\end{array}$ \\
\hline RT & ShKh 15 & Stage 3 & 21,278 & $1,9 \%$ & \multirow{6}{*}{ Unidirectiona } \\
\hline TS-1 & ShKh 15 & Stage 2 & 0,296 & & \\
\hline TS-1 & ShKh 15 & Stage 3 & 19,421 & & \\
\hline $\mathrm{AOH}-10$ & ShKh 15 & Stage 3 & 51,76 & $2,5 \%$ & \\
\hline TS-1* & ShKh 15 & Stage 2 & 1,98 & & \\
\hline TS-1* & ShKh 15 & Stage 3 & 19,58 & & \\
\hline Vaseline oil & Steel 45 & Stage 3 & 8,96 & & Bidirectional \\
\hline
\end{tabular}

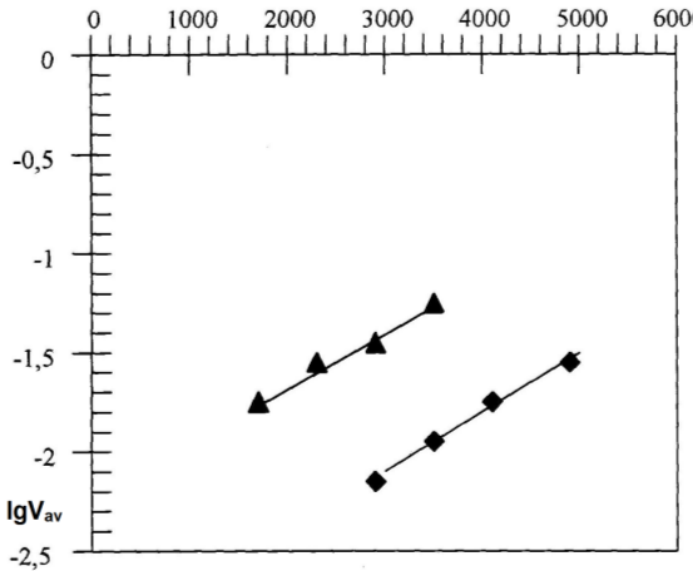

Fig. 2. The average total wear logarithm of three balls $\lg \mathrm{V}_{\mathrm{av}}$ and the duration $\mathrm{t}$ for steel ShKh 15 in long-term storage of TS-1 aviation fuel.

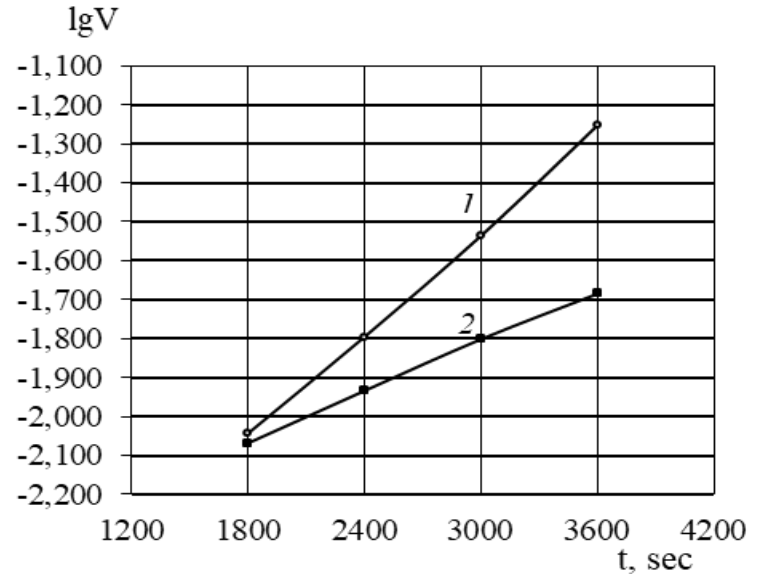

Fig.3 . The average total wear logarithm of three layers and the duration of tribo-kinetic experiments for fuel TS-1 when 1-T = $333 \mathrm{~K}$; and $2-\mathrm{T}=303 \mathrm{~K}$. 
According to the results that obtained by the experiment in (Table 1,2) the (Figure 2) and (Figure 3) were constructed.

With using the proposed methodology for assessing the kinetic characteristics, the values of the activation energy for two stages of triboreaction and a number of lubricants have been obtained in ( Table 3).

The universality of the activation energy $E$ lies in the fact that this energetic, integral, materials science criterion for assessing three stages of triboreaction is applicable for assessing both the wear resistance of structural materials and wear-resistant fuels and lubricants. This is achieved by studying the materials of triboconjunction: when we change the structural material with a fixed lubricant, then the Eact value characterizes the wear resistance of the structural material under study. And vice versa: by changing lubricants with a fixed structural material, we determine the wear-resistant properties of the lubricants under study. This procedure was performed in the present work.

\section{CONCLUSION}

1. The possibility of kinetic description of the normal mechanic-chemical wear of the structural elements of tribo-conjugations at boundary lubrication as a general threestage tribological reaction (triboreaction) has been experimentally confirmed.

2. The experimental calculated method of determination of the kinetic and energyactivation criteria for the evaluation of the wear resistance and compatibility of tribomaterials was experimentally confirmed on the basis of kinetic three-stage triboreaction.

3. The criteria for the evaluation of wear resistance and compatibility of rubbing materials based on the developed methodology for determination of kinetic and energy-activation as well as for evaluation of activation energies of the II and III stages of the triboreaction for determination of the anti-wear properties of long-term storable aircraft fuels TS- 1 and TS$1^{*}$ and also for determination of the activation energy of wear and tear for Steel
45 during reciprocating motion have supplemented the matrix energy-activation criterion for the evaluation of the wear resistance and compatibility of tribomaterials.

4. By to the proposed matrix energy-activation criterion for assessment of the durability and compatibility of triboconjunction materials it is possible to control the durability and compatibility of triboconjunction materials due to the advantage of either one or two stages of tribo reaction.

5. The obtained values $\left(E^{d}\right)$ of anti-wear properties of aircraft long-term storable jet fuels TS-1 and TS- $1^{*}$ and $\left(E^{d}\right)$ of Steel 45 during reciprocating motion can be used to accumulate the bank of energy activation criteria for the evaluation of the durability and compatibility of tribomaterials.

\section{REFERENCES}

[1] O.I. Bogdanovich, Practical use of activation energy of wear resistance and compatibility of tribo-couple materials, in Proceedings of the VIII world congress Aviation in the XXI st CenturySafety in Aviation and Space Technologies, 1012 October, 2018, Kyiv, Ukraine, pp. 27-29.

[2] B.I. Kostetskiy, Fundamental patterns of friction and wear, Publishing house Knowledge, USSR, 1981. (in Russian)

[3] V.V. Fedorov, Thermodynamic aspects of strength and fracture of solids, Tashkent: Fan Uz. SSR, 1979. (in Russian)

[4] N.A. Bushe, V.V. Kopyitko, Compatibility of rubbing surfaces, Publishing house Science, 1981, (in Russian)

[5] P.V. Nazarenko, L.I. Bershadskiy, A.Y.A. Shepel, O.I. Bogdanovich, Energy-activation regularity of the structural adaptability of ShKh-15 steel, Problems of friction and wear, Inter disciplinary Scientific and Technical publication, iss. 26, pp. 3-8, 1984 (in Russian).

[6] L.I. Bershadskiy, A.I. Bogdanovich, Experimental study of the invariance of the activation energy of wear of ShKh-15 steel in the range of structural adaptability, in II All-Union Scientific and Technical Conference Tribotechnics - mechanical engineering, November, 1983, pp. 179-180. (in Russian).

[7] O.I. Bogdanovich, The experimental determination of the activation energy of wear in 
a hydrocarbon environment, Problems of friction and wear, Inter disciplinary Scientific and Technical publication, iss. 19, pp. 34-38, 1981, (in Russian).

[8] F.F. Vitman, N.A. Zlatin, L.M. Shestopalov, The Relation between the activation energy of metals and their resistance to deformation, in Conference dedicated to the 70th anniversary of academician A.F. Yoffe, 1950, pp. 331-340. (in Russian).

[9] V. Arono, Formation and destruction kinetics of transformed structures of metals in frictional contact, Wear, vol. 38. iss 2, pp. 305-310, 1976, doi: 10.1016/0043-1648(76)90078-8

[10] V. Arono, Kinetic characteristics of the transformation and failure of the surface layers of metals under dry friction, Wear, vol. 41, iss 2, pp. 205-212, 1977, doi: 10.1016/00431648(77)90001-1

[11] I.A. Buyanovskiy, N.V. Gittis, A.G. Ginzburg, Method for determining the activation energy of the film starvation process, in II All-Union Scientific and Technical Conference Tribotechnics - mechanical engineering, November, 1983, pp. 1080-1081, (in Russian).

[12] I.A. Buianovskyi, Development of a temperaturekinetic method for determining the characteristics of the boundary lubrication process and choice of lubricants, PhD thesis, Moscow, pp 44, 1989, [In Russian].

[13] V. P. Tikhomiro, O. A. Horlenko, Kinetic model of friction of solids, Friction and Wear, vol. 21. no. 2, pp. 121-127, 2000, [In Russian].

[14] V.G. Melnikov, Influence of friction parameters on the activation energy of tribo-chemical decomposition of lubricants in the rolling friction zone, Friction and Wear, vol. 22, no. 5, pp. 567574, 2001, [In Russian].

[15] A.I. Neko, Analysis of Cavitation-erosion wear as a process of corrosion-mechanical destruction, Friction and Wear, vol. 5, no. 4, pp. 748-753, 1984, (in Russian).

[16] 0.0. Mikosyanchy`k, Structurally energetic and rheological indicators of the lubricating layer in the friction contacts under conditions of unstable operating modes, PhD thesis, Kyiv, 2017, (in Russian).

[17] A.K. Dedkov, Investigation of the interaction of the gas environment and the friction solids surface (in relation to the friction nodes of the PTM), PhD thesis, 1975, (in Russian).

[18] R.M. Matveevskiy, I.A. Buyanovskiy, V.L. Lishhi, A.B. Vipper, Evaluation of the activation energy of the process of chemical modification of friction under conditions of boundary lubrication, Chemistry and technology of fuels and oils, vol. 2, pp. 50-52, 1976, (in Russian).

[19] B.I. Kostetski, L.I. Bershadskiy, E.N. Chukreev, The phenomenon of self-regulation in the metals wear, Report, USSR Academy of Sciences vol. 191, no. 6, pp. 1339-1342, 1970, (in Russian).

[20] O.I. Bogdanovich, Activation energy of anti-wear properties of TS-1* long-term collection fuel, in the IV International conference of NAU, 23-25 April, 2002, NAU, pp. 43-59, (in Ukraine).

[21] O.I. Bogdanovich, Kinetic and energeticactivation characteristics of wear resistance and compatibility of materials of tribo-conjugation, PhD thesis, Kyiv, 1987, (in Russian).

[22] O.I. Bogdanovych, Comparison of the wear activation energy ( $\left.E^{d}\right)$ ShKh15 in fuel TS-1 of a long-term storage, in Proceedings of the Third World Congress Aviation in the XXI-st century, 22-24 September, 2008, National Aviation University, Kyiv, pp. 11.25-11.27.

[23] O.I. Bogdanovich, Estimation of opposite properties of TS-1 aviation fuel with the help of wear activation energy, in Proceedings of the Agrotechnical Academy State, Melitopo, 2006, vol. 39, pp. 80-84, (in Ukraine).

[24] O.I. Bogdanovich, Technology for assessing the energy activation of mechano-chemical modification of ShKh15 steel in carbohydrate environment, in the Technical conference on Materials in science , 23-25 April, 2003, NAU, pp. 43-73, (in Ukraine).

[25] O.I. Bogdanovich, Estimation of kinetic characteristics and modification activation energy of ShKh15 in aviation fuel TS-1, in Proceedings of the VI World Congress Aviation in the XXI st Century - Safety in Aviation and Space Technologies, 23-25 September, 2014, Kyiv, NAU, pp 1.6.9-1.6.13.

[26] O.I. Bohdanovych, V.V. Tokaruk, Yu.Ya. Statnikov, Determination of activation energy of mechano-chemical modification of ShKh15 steel in the medium of long-term- stored aviation fuel TS-1, Problems of Friction and Wear, vol. 86, no. 1, pp. 93-97, 2020, [In Ukrainian].

[27] O.I. Bogdanovich, I.V. Tkachenko, K.E. Grinkevich, Estimation of kinetic characteristics and activation energy of wear during reciprocating doubling, in The International scientific and technical conference, Materials in Avia-2009, 21-23 September, 2009, Kyiv, pp. 145-148, (in Russian). 